\title{
¿Es posible diferenciar analíticamente un consumidor de coca de uno de cocaína?
}

\author{
Franklin Alcaraz del Castillo*; Julia ZuazoY.* \\ * Centro Latinoamericano de Investigación Científica, La Paz, Bolivia \\ Enviar correspondencia a: \\ Franklin Alcaraz Del Castillo, casilla 5243, La Paz Bolivia. celin@celinbolivia.org
}

\section{RESUMEN}

El objetivo del estudio fue ver si a través del análisis de orina se podría determinar el tipo de consumo de coca o cocaína que realizaban los consumidores. El estudio se llevó a cabo en la ciudad de La Paz (Bolivia). Para ello se reclutaron consumidores voluntarios y se dividieron en tres grupos: los consumidores de coca masticada (acullicadores), los consumidores de cocaína y los consumidores de coca en forma de mates. A todos ellos se les tomó muestras de orina con el fin de detectar la cantidad de benzoilecgonina y verificar si se podían establecer diferencias entre los individuos o los grupos.

Cuando las concentraciones de benzoilecgonina en orina fluctuaban entre 220 a $450 \mathrm{ng} / \mathrm{ml}$ correspondían a cualquiera de los tres grupos consumidores sin poder descartar, ni diferenciar, ninguna de estas posibilidades.

Cuando las concentraciones de benzoilecgonina en orina eran más altas y fluctuaban entre 451 a 560 ng/ml, correspondían a consumidores de hoja de coca que la masticaban ("acullicaban") o a los consumidores de cocaína, sin poder descartar, ni diferenciar, ninguna de las dos posibilidades.

La eliminación de benzoilecgonina por la orina, depende de la cantidad de coca/cocaína ingerida, de la forma de consumo de la coca, del metabolismo de la persona y de la cantidad de líquidos ingeridos/eliminados; pero no fue posible mediante esta prueba, saber a qué grupo pertenecía un individuo, pero sí se pudieron establecer diferencias grupales significativas entre las distintas formas de consumo.

Palabras clave: consumidores de cocaína, tomadores de mate, masticadores de hoja de coca, benzoilecgonina, determinación en orina.

\section{ABSTRACT}

The objective of the study was to ascertain if urine analysis could be used to determine whether substance users were taking coca or cocaine. The study was carried out in the city of La Paz in Bolivia. Volunteer users were recruited and divided into three groups: those who chewed coca leaves, cocaine users and coca tea drinkers. Urine samples were taken from all of them, with the objective of detecting the amount of benzoylecgonine, and ascertaining if differences could be established between individuals or groups.

When benzoylecgonine concentrations in urine fluctuated between 220 and $450 \mathrm{ng} / \mathrm{ml}$, they corresponded to users in any of the three groups, it being impossible to discard or differentiate any one of them.

When benzoylecgonine concentrations in urine were higher, and fluctuated between 451 and $560 \mathrm{ng} / \mathrm{ml}$, they corresponded to those who chewed coca leaves or to cocaine users, without it being possible to discard or differentiate either of these two groups.

The elimination of benzoylecgonine in the urine depends on the quantity of coca/cocaine ingested, the cocaine consumption method, personal metabolism and the quantity of liquid ingested/ eliminated but, on the basis of this study, it was not possible to ascertain to which group an individual belonged, although it was possible to establish significant group differences in accordance with the specific methods of use.

Key words: cocaine users, coca tea drinkers, coca leaf chewers, benzoylecgonine, determination in urine.

La hoja de coca es un arbusto que crece en un ambiente ecológico húmedo, entre los 500 y 2000 metros sobre el nivel del mar. Pertenece al género de la familia de plantas tropicales Ilamadas Erythroxyláceas. Existen 250 especies de esta planta en todo el planeta. En América crecen alrededor de 200 especies. De las 250 que se encuentran en el mundo, solo cuatro son "domésticas" y las cuatro son americanas. ha sido la motivación principal del presente estudio. 
El resto son plantas silvestres. Tradicionalmente la hoja de coca que se consumía lícitamente en Bolivia es la "Erythroxylon coca var coca". Se la llama también "Coca Boliviana", aunque también se la cultiva en el Perú, donde recibe el nombre de "Huanuco". La segunda especie de coca es la variedad "Ipadu" (llamada "Epadú" por los brasileños), que crece en la región amazónica. La tercera clase es la "Erythroxylon Novogranatense" variedad "Novogranatense" que se desarrolla en Colombia y por lo que es conocida como "Coca Colombiana." Finalmente la variedad "Truxillense" de la "Erythroxylum Novogranatense", es la cuarta variedad doméstica y debe su nombre a la región homónima del Perú donde se la cultiva (Trujillo), aunque crece también, en forma reducida, en el norte ecuatoriano. La zona tradicional de cultivo de este arbusto en Bolivia, es los Yungas del departamento de La Paz, donde se cultivaba coca desde un período anterior a la conquista española. La hoja de coca siempre ha tenido usos lícitos ${ }^{1}$. Hasta antes de la década de los años setenta, se la usaba como medicamento (mates y emplastos), para ritos místicos (adivinaciones, ofrendas a la madre tierra o "Pachamama", mal de amores, etc.) y para el "acullico" 2 . El mate también se lo bebe - aún hoy - de manera recreacional. A principios de la década de los setenta sin embargo, los cultivos de esta planta empezaron a crecer explosivamente fuera de las áreas tradicionales de cultivo, en la región del Chapare del departamento de Cochabamba. Esta producción se destinó y se destina a la fabricación de cocaína, droga fuertemente estimulante que crea dependencia (Alcaraz, 1989).

En Bolivia y Perú, pocos parecen darse cuenta que el consumo de hoja de coca - especialmente en forma de "acullico" - no es universal, sino que más bien es la excepción si consideramos el consumo a escala mundial. Solo en estos dos países es todavía lícito este consumo y aunque en Colombia todavía hay sectores de la población que "mambean" 3 este vegetal, su magnitud, trascendencia cultural y social no alcanza a la que tiene en Bolivia y Perú. Dentro de la discusión que permanece abierta, han sido los deportistas, especialmente los jugadores de fútbol, los que de alguna manera han puesto (y lo siguen haciendo) el tema de actualidad, cuando sus exámenes antidoping, en diferentes oportunidades han dado positivo, y ellos alegaban haber consumido mate de hojas de coca y no cocaína. Hasta donde sabemos, solo en Perú se intentó diferenciar este consumo (Zavaleta, De La Mata, Sin fecha). En Bolivia, el presente estudio es el único intento. La pregunta que surge es ¿por qué es importante diferenciar este consumo? Porque en esta parte de América todavía hay un importante sector de población que utiliza la hoja de coca para consumirla lícitamente.

De acuerdo al Centro Latinoamericano de Investigación Científica (Alcaraz, Soliz, Zuazo, 2000) alrededor del $14 \%$ de la población boliviana actual, es decir más de un millón de personas, todavía consume hoja de coca en sus diferentes formas. Bolivia es también un país donde se produce hoja de coca en forma legal y aunque la Ley 1008 delimita claramente las áreas de cultivo autorizadas, todavía existen plantaciones que el gobierno boliviano ha denominado "áreas de cultivo excedentarias", cuya producción estaría destinada a la fabricación de cocaína, Este hecho - y el que no se pueda diferenciar al consumidor lícito de hoja de coca del de cocaína- es el antecedente que hace necesario diferenciar el uso lícito del ilícito de la hoja de coca, es decir, separar el consumo de la hoja de coca como tal del de la cocaína. Para abundar más en el tema: la pregunta fue ¿cómo diferenciar un individuo que consume(ió) coca, en sus diferentes formas de uso lícito, de uno que consume(ió) cocaína? El hecho de que la producción de la hoja de coca en Bolivia esté fuertemente relacionada a una economía de subsistencia en las zonas productoras, ha creado las condiciones para que el tema se convierta en político y siente las bases para la creación de un partido que hoy interviene del Congreso Boliviano, con representantes de extracción indígena. Estos nuevos legisladores, muchos de ellos consumidores de la hoja de coca, la defienden a pesar de saber que este arbusto es también materia prima para la fabricación de cocaína. Por lo tanto, el gran desafío fue - y es - llevar a cabo estudios capaces de aclarar el tema. Y es el planteamiento de la presente investigación. Los resultados de la misma, sin embargo, han terminado por mostrar un panorama ampliado que necesita de mayores estudios y a los que deben dedicarse tiempo y recursos.

\footnotetext{
1 El uso lícito de la hoja de coca esta apoyado por la Convención de Viena referida al tema y la Ley 1008 del Estado Boliviano que delimita zonas de cultivo de la planta de coca "destinadas al uso tradicional" (lícito) y que especifica como una superficie de 12.000 hectáreas en los Yungas de la Paz y Mandiola de Cochabamba. Toda la producción que no este dentro de lo especificado por la Ley 1008, es considerada ilegal, por lo tanto, sujeta a "erradicación".

2 "Acullicar" es un término quechua/aymara. Se lo utiliza para designar al usuario de la hoja de coca. El buen "acullicador" introduce la hoja de coca, secada al sol, sin ningún proceso adicional, y la va depositando en los carrillos de la boca hasta formar un bolo que suele mezclarse con una substancia alcalina llamada generalmente "Llijta" (aunque también recibe otros nombres), que remoja con la saliva y de la que va extrayendo el jugo en un proceso que puede durar todo el día.

${ }^{3}$ El "mambeo" colombiano tendría que ser el equivalente del acullico boliviano o chajchado peruano, con la diferencia que el "mambeador" mastica la hoja de coca.
} 


\section{LA COCA Y LA COCAÍNA}

De acuerdo a la literatura existente (Instituto Boliviano de Biología de la Altura, 1997), es indiscutible el hecho de que tanto la hoja de coca seca, que se utiliza para fabricar mate en bolsitas, sola o acompañada de anís, manzanilla o cualquier otra substancia, produce los mismos metabolitos que la cocaína ingerida en forma de clorhidrato o pasta base, es decir:

\section{Ecgonina \\ Metilecgonina \\ Benzoilecgonina y \\ Norformilecgonina ${ }^{4}$}

En el presente estudio, uno de estos metabolitos, la benzoilecgonina, fue objeto de detección por parte del laboratorio, en un proceso que intentó diferenciar concentraciones de benzoilecgonina de acuerdo a la cantidad y calidad (forma de consumo) de la hoja de coca ingerida.
El objetivo general del estudio consiste en explorar indicadores cuantitativos para diferenciar el consumo de hoja de coca y de cocaína.

\section{MATERIALY MÉTODOS}

El organismo operativo del presente estudio, fue el Centro Latinoamericano de Investigación Científica, más conocido por su sigla de CELIN BOLIVIA, con la contratación de "Laboratorios La Paz" en la parte de los análisis de orina. El estudio se llevó a cabo en la ciudad de La Paz, Bolivia observando las siguientes etapas:

- Se reclutaron voluntarios para que consuman mate de coca en sus dos formas: en bolsita y en hoja de coca (sin proceso alguno excepto su secado al sol) y voluntarios "acullicadores" 5 consuetudinarios de hoja de esta planta, para tomarles muestras de orina donde se hizo detección de benzoilecgonina.

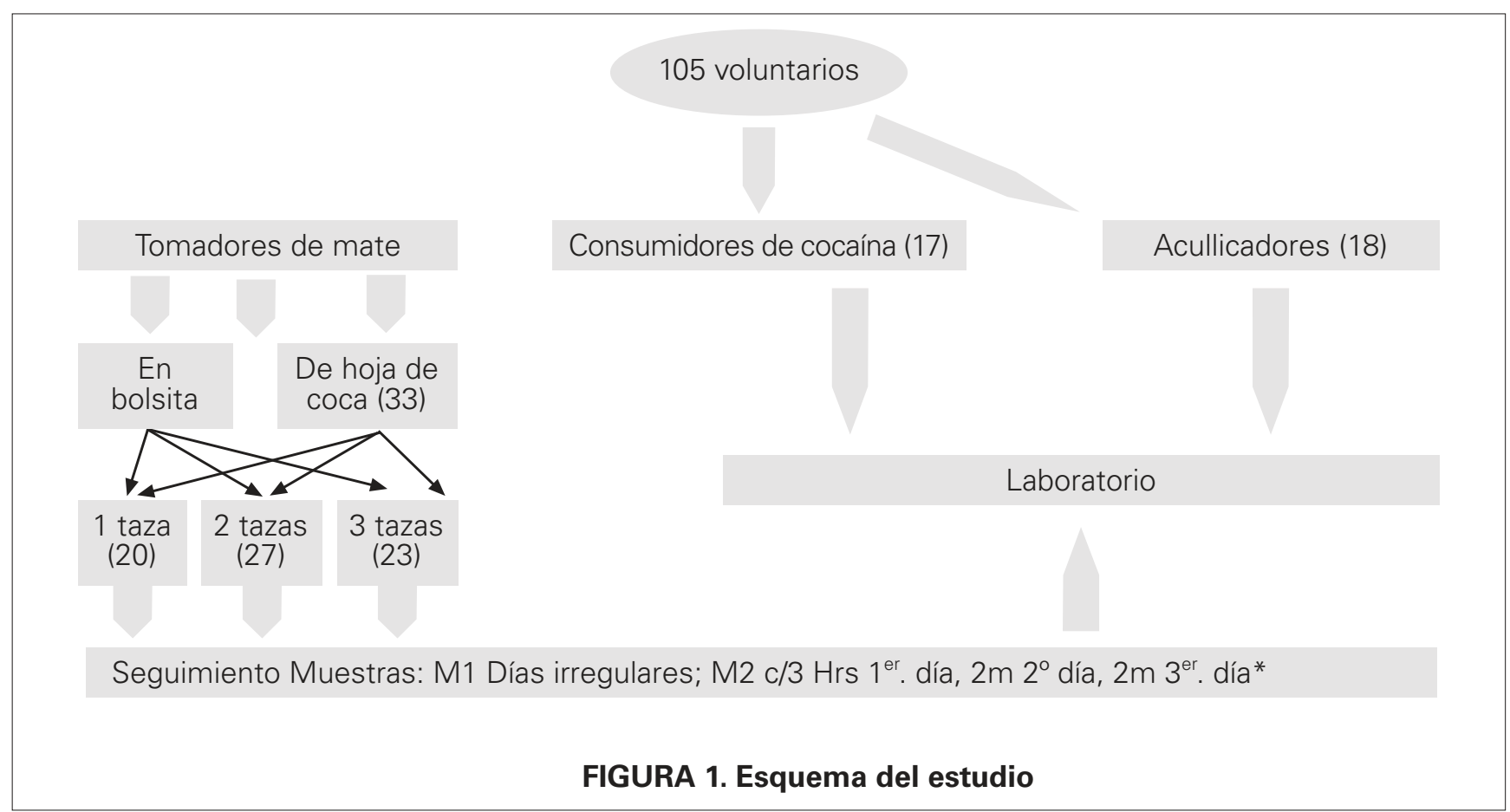

M1 = Grupo muestral de tres personas que bebieron mate de coca a las que se tomó muestras de orina de manera irregular (dos el primer día y luego una matutina el segundo y el tercer día y se repitió una toma de muestra el 5to. Día en la mañana). Entre paréntesis figura el número de voluntarios para cada forma de consumo.
M2= Grupo muestral mayoritario de bebedores de mate (en diferentes formas según se observa en el esquema) al que se tomó muestras de orina cada tres horas el primer día y dos muestras el 2 do. y 3er. día (una en la mañana y otra en la tarde con un intervalo de 12 horas entre una y otra).

\footnotetext{
${ }^{4}$ Hernán Osorio, de la Universidad de Chile, se encuentra trabajando en un nuevo metabolito: la metahidroxibenzoilecgonina (Ver la página web de la Universidad de Chile: www.uchile.cl/undin2/actuales/noti2826.shtml).

5 "Acullicador" es la persona que "Acullica" (Ver referencia número 2).
} 
- Se reclutaron voluntarios consumidores de cocaína (como pasta base o clorhidrato) también para recolectar muestras de orina y cuantificar el contenido de benzoilecgonina en las muestras obtenidas.

- Se conformó el equipo central de investigadores.

- Se efectuaron sesiones de toma de mate de coca y recolección de muestras de orina.

- Un grupo del equipo central viajó al altiplano boliviano (alrededores del lago Titicaca), para reclutar voluntarios "acullicadores" de hoja de coca indígenas, de los que también se recolectaron muestras de orina.

- Se efectuaron los correspondientes análisis de las muestras de orina

Tal como se muestra en el esquema de la investigación (Ver figura 1), se tomaron 105 voluntarios a los que se les dividió en tres grupos:
- Tomadores de mate hecho a partir de hojas de coca o del industrializado en forma de bolsitas de té (de una taza, dos tazas y tres tazas, voluntarios).

- Masticadores de hojas de té o "acullicadores" (Población indígena del altiplano bolivia no, voluntarios)

- Consumidores de cocaína (Drogodependientes voluntarios)

Los voluntarios consumidores de cocaína, eran drogodependientes que se prestaron a colaborar a cambio de no divulgar sus nombres, direcciones ni formas de identificación y/o localización alguna. Su consumo de cocaína, en forma de pasta base y/o clorhidrato, antes de la toma de muestra, fue de entre $1 \mathrm{y}$ 5 gramos de cocaína en 24 horas $^{6}$

Las muestras se enviaron al laboratorio a medida que se obtenían, donde se continuaba con el siguiente esquema:

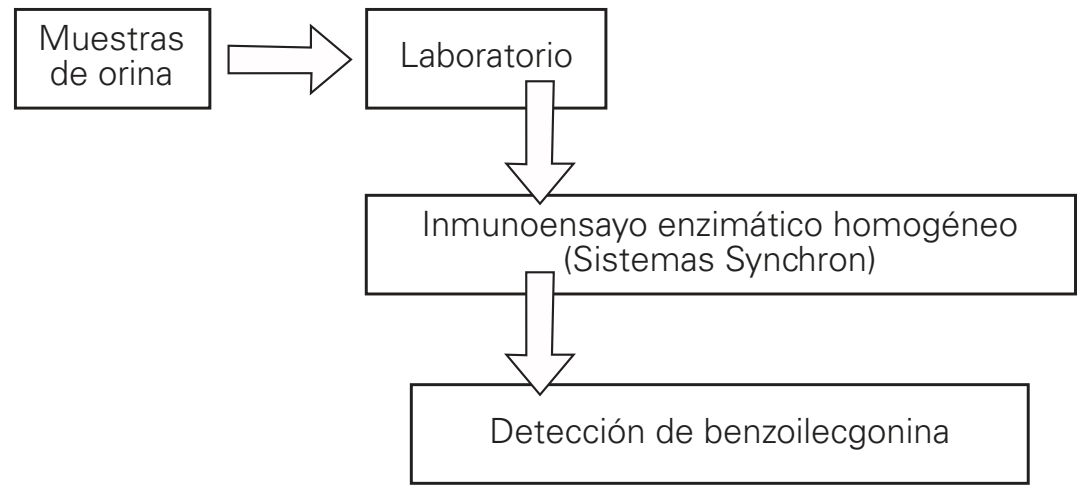

Figura 2. Esquema del proceso en laboratorio

Una vez obtenido el informe del laboratorio, este se revisó, clasificó y se introdujo a la base de datos elaborada en la oficina central del CELIN en programa MICROSOFT ACCESS para su proceso e interpretación posterior en programa EPI-INFO.

Finalmente, al cumplimiento del trabajo de campo y laboratorio, se elaboró el presente informe.

\section{RESULTADOS}

La tabla 1 muestra el número de muestras de orina aportadas por los voluntarios para las diferentes formas de consumo de hoja de coca y cocaína. Muestra también la edad media de los voluntarios, así como la edad mínima y la edad máxima de los mismos en cada una de las categorías.

\footnotetext{
${ }^{6}$ No se pudo establecer con precisión la cantidad de droga que cada uno de los voluntarios había utilizado, debido a que, en el proceso de consumo, los drogodependientes no son conscientes del mismo y muchos de ellos utilizan pasta base y clorhidrato al mismo tiempo (se les pidió expresamente no usar otras drogas) o en períodos secuenciales. Por otra parte, no permitieron la presencia de un miembro del equipo de investigadores y además suelen desplazarse de un lugar a otro y muchos de ellos eran internos del penal de San Pedro de La Paz, de modo que tuvimos que aceptar sus propias apreciaciones después de mostrarles cantidades de leche en polvo o pasta de papel maché para apreciar las cantidades estimadas en el presente estudio. Finalmente también hay que considerar que las cantidades ingeridas de cocaína, estaban "estiradas", es decir, mezcladas en diferente proporción con otras substancias como yeso, tiza, talco, leche en polvo, etc.
} 
TABLA 1. Detalle de voluntarios según edad y forma de consumo

\begin{tabular}{|lccccc|}
\hline Forma de consumo & Muestras & Edad media & Desviación estándar & Edad mínima & Edad máxima \\
\hline Mate en bolsitas & 37 & 36,1 & 9,2 & 20 & 60 \\
Mate en hojas & 28 & 38,8 & 10,4 & 21 & 62 \\
Acullico & 20 & 54,1 & 16,6 & 20 & 78 \\
Consumidores cocaína & 20 & 35,4 & 7,5 & 25 & 49 \\
Total & 105 & 40,1 & 12,9 & 20 & 78 \\
\hline
\end{tabular}

Es importante analizar cuidadosamente la información de la tabla número dos para poder distinguir las diferencias que se dan en la concentración de benzoilecgonina en orina provenientes de las diferentes formas de consumo de la coca-cocaína, en nanogramos por mililitro (ng/ml). Es importante ver la media y los valores máximos y mínimos.

Lo que nos muestra la tabla 2, es que los consumidores de cocaína tienen un rango más amplio de concentración de benzoilecgonina en orina que los otros grupos (entre los valores máximo y mínimo), pero la media de los consumidores de cocaína, aunque pare- ce ligeramente inferior a la media de los "acullicadores", estadísticamente no lo es, porque si observamos el intervalo de confianza (al $95 \%$ ) para la media mostrado en la tabla 2- el análisis nos enseña que no hay diferencia estadísticamente significativa entre la media de los consumidores de cocaína y los consumidores de hoja de coca en forma de acullico.

Es decir que los valores medios para los "acullicadores" y para los consumidores de cocaína, son prácticamente iguales.

Los demás si muestran diferencias en los valores medios de consumo.

Tabla 2. Estadísticas descriptivas del nivel de concentración de benzoilecgonina en orina

\begin{tabular}{|lccccccc|}
\hline & & \multicolumn{7}{c|}{$\begin{array}{c}\text { Intervalo al 95\% de } \\
\text { confianza para la media }\end{array}$} \\
\cline { 5 - 7 } Forma de consumo & Muestras & Media & $\begin{array}{c}\text { Desviación } \\
\text { estándar }\end{array}$ & $\begin{array}{c}\text { Límite } \\
\text { inferior }\end{array}$ & $\begin{array}{c}\text { Límite } \\
\text { superior }\end{array}$ & Mínimo & Máximo \\
\hline Mate en bolsitas & 37 & 365,2 & 44,2 & 350,5 & 379,9 & 295 & 447 \\
Mate en hojas & 28 & 334,8 & 48,7 & 315,9 & 353,7 & 235 & 394 \\
Acullico & 20 & 395,4 & 27,1 & 382,7 & 408,1 & 351 & 458 \\
Consumidores cocaína & 20 & 391,1 & 44,8 & 370,2 & 412,0 & 344 & 557 \\
Total & 105 & 367,8 & 48,3 & 358,4 & 377,1 & 235 & 557 \\
\hline
\end{tabular}

La figura 3 es más clara en lo mencionado anteriormente porque nos muestra en forma de barras las diferencias de los valores medios de consumo de la coca-cocaína en sus diferentes modalidades. En otras palabras: los tomadores de mate de coca forman un grupo que se diferencia claramente del otro grupo formado por "acullicadotes" y consumidores de cocaína, (asumiendo que consumieron el mismo día). Por lo tanto, no es posible diferenciar, en el presente estudio, entre quienes acullican y los que consumen cocaína. Pero el equipo de investigación tenía dudas sobre si la cantidad ingerida de mate de coca, en sus dos formas, influía en la concentración de benzoilecgonina en orina. Como se contaba con voluntarios que habían bebido hasta tres tazas de mate de coca, no fue difícil comparar los resultados.

Lo primero que llamó la atención es que, en todos los casos, los bebedores de mate en bolsitas tenían concentraciones superiores de benzoilecgonina en orina que los bebedores de mate de coca hecho de hojas de coca, no obstante la evidente menor cantidad de hoja de coca en bolsitas ( 2 a 3 gr.) que la cantidad de hojas enteras remojadas (entre 6 a 15 enteras, es decir 5 a $10 \mathrm{gr}$.). El análisis posterior llevó a concluir que la hoja de coca triturada o molida, liberaba mayor cantidad de benzoilecgonina.

Lo segundo es que, de acuerdo a la figura número cuatro, hay diferencia fácilmente apreciable entre 


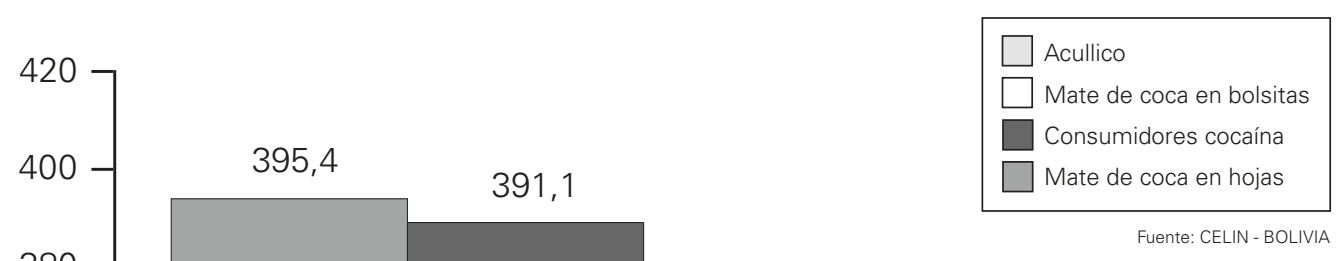

365,2

300

Figura 3. Promedio de concentración de benzoilecgonina en orina (Mates de coca, "acullico", consumidores de cocaína.

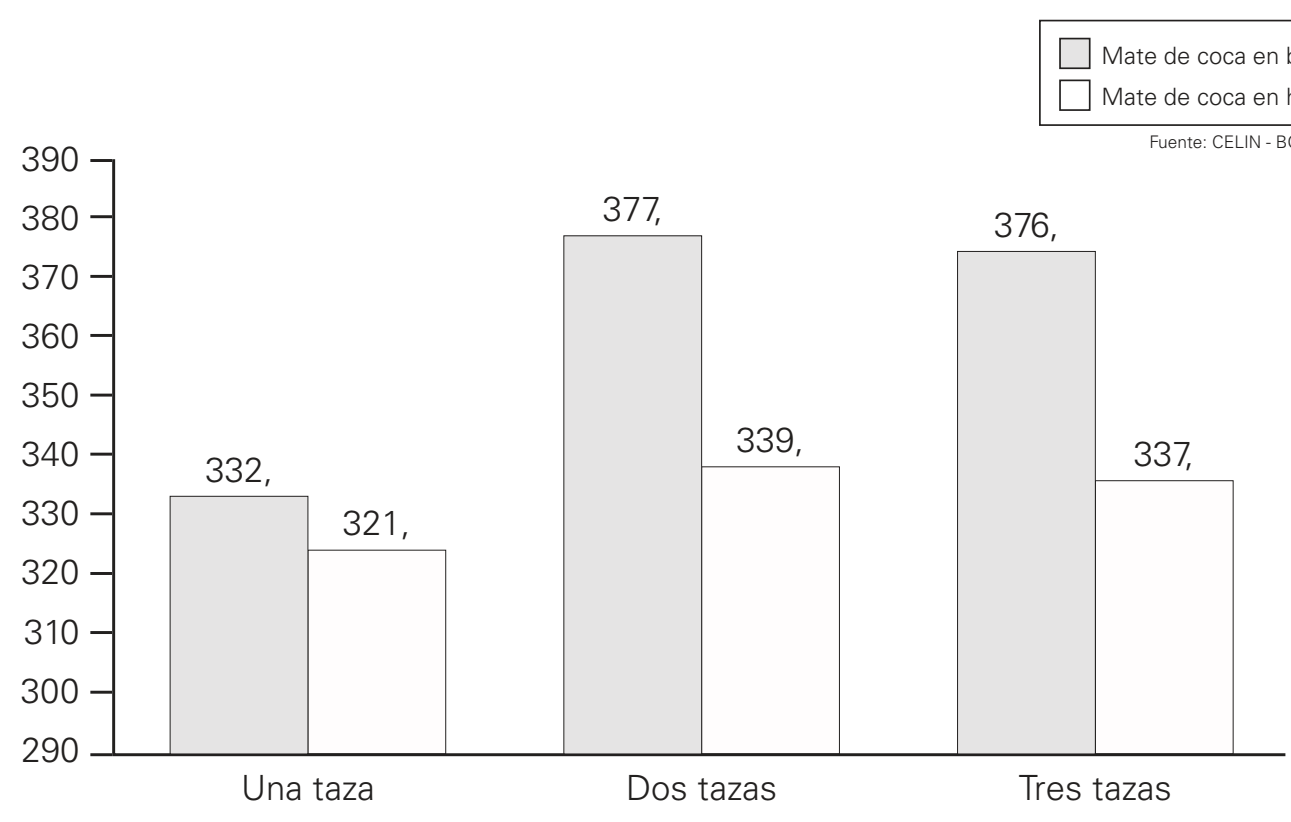

Figura 4. Promedio de concentración de benzoilecgonina en orina según número tazas de mate de coca ingeridas.

los tomadores de una taza de mate y aquellos que beben dos o más; pero no hay diferencia entre los que beben dos o tres tazas. Es decir, las concentraciones de benzoilecgonina en orina entre aquellos que beben dos o tres tazas de mate de coca, son iguales. Solo se diferencian en cuanto a los bebedores de mate de coca en bolsitas con los bebedores de mate de hoja de coca entera.

\section{DETECCIÓN DE BENZOILECGONINA EN ORINA DE TOMADORES DE MATE DE COCA}

Por otra parte, aunque la literatura disponible menciona que la detección de benzoilecgonina en orina de drogodependientes consumidores de cocaína se hace positiva entre los 30 a 45 minutos de la administrada 
la droga y permanece positiva por 72 horas (término medio), decidimos comprobar si ocurre lo mismo con la hoja de coca (mates).

La figura 5 podría prestarse a discusión si no se conocieran los antecedentes para su elaboración. Se la realizó con tres voluntarios que tomaron mate de coca (para el caso no importa la forma) cuando una de las hipótesis formuladas antes del estudio señalaba un probable tiempo de excreción de benzoilecgonina mayor a tres días para los tomadores de mate de esta substancia.

Los voluntarios bebieron mate de coca y aportaron con una muestra el primer día, y otra el segun- do; a partir del tercero y cuarto día no se recolectaron muestras. El quinto día se recolectó una muestra de orina que resultó negativa en los tres voluntarios. El voluntario que dio negativo el segundo día (Línea con cuadrados), era gran bebedor de agua.

Sin embargo, todavía se tenía que comprobar o rechazar las hipótesis mencionadas anteriormente. Para hacerlo, tomamos y analizamos muestras de orina por horas, como se muestra en la siguiente figura (Número 6).

Aquí lo interesante es observar que las diferentes concentraciones de benzoilecgonina fluctúan, para uno de los casos (línea de cuadrados) durante ocho

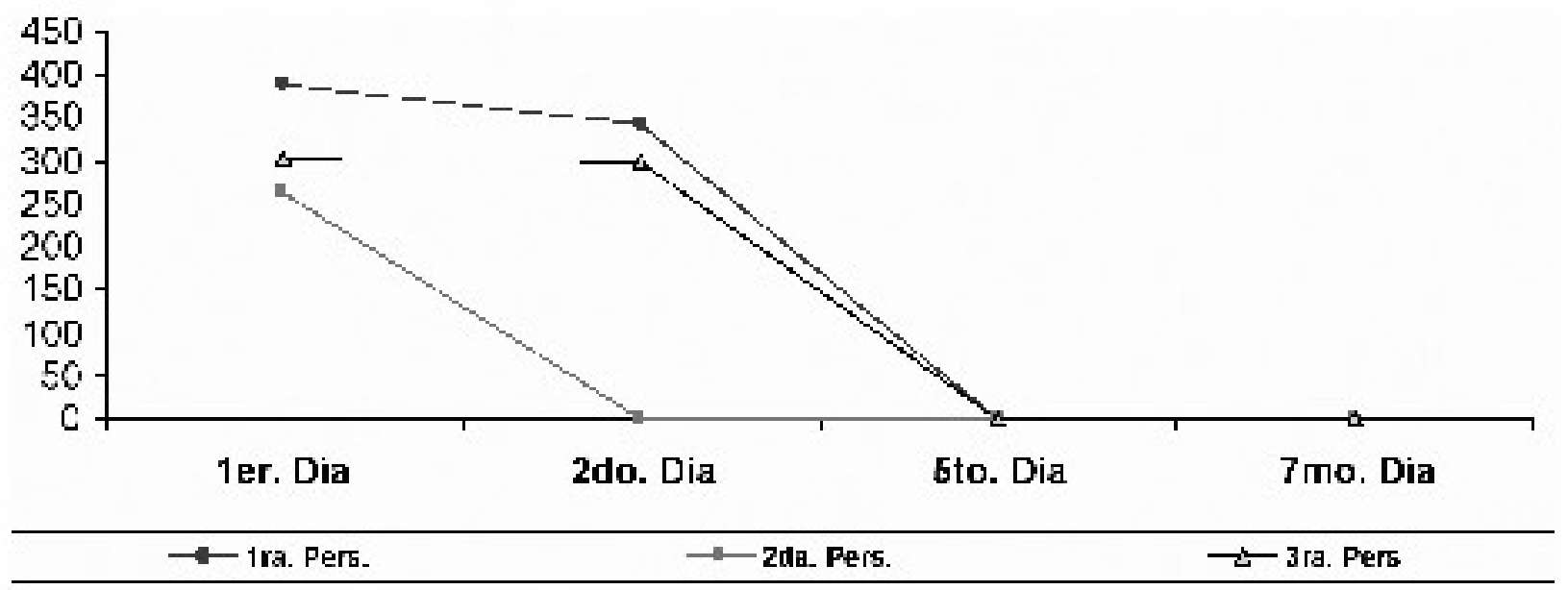

Figura 5. Concentración de benzoilecgonina en muestras de orina, según días de recolección (personas que consumieron 3 tazas de mate de coca en hojas)

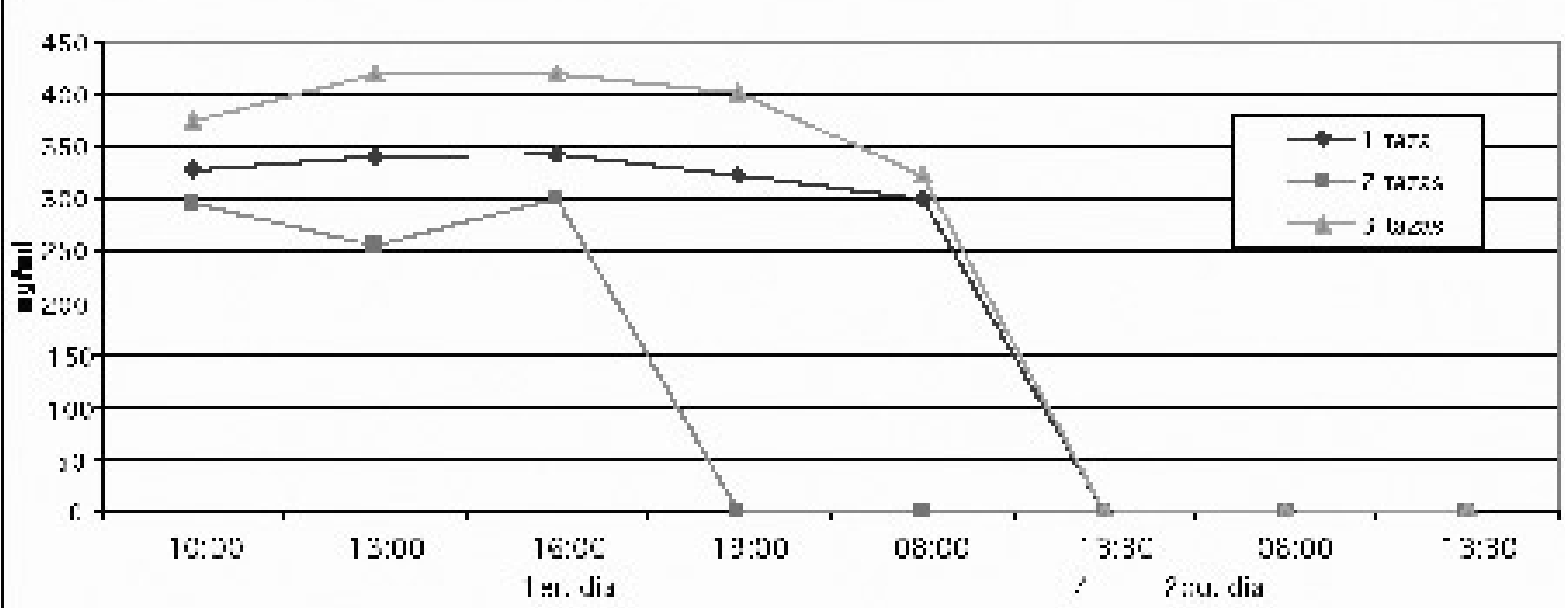

Figura 6. Concentración de benzoilecgonina en muestras de orina según excreción por horas (Consumidores de mate de coca.) 


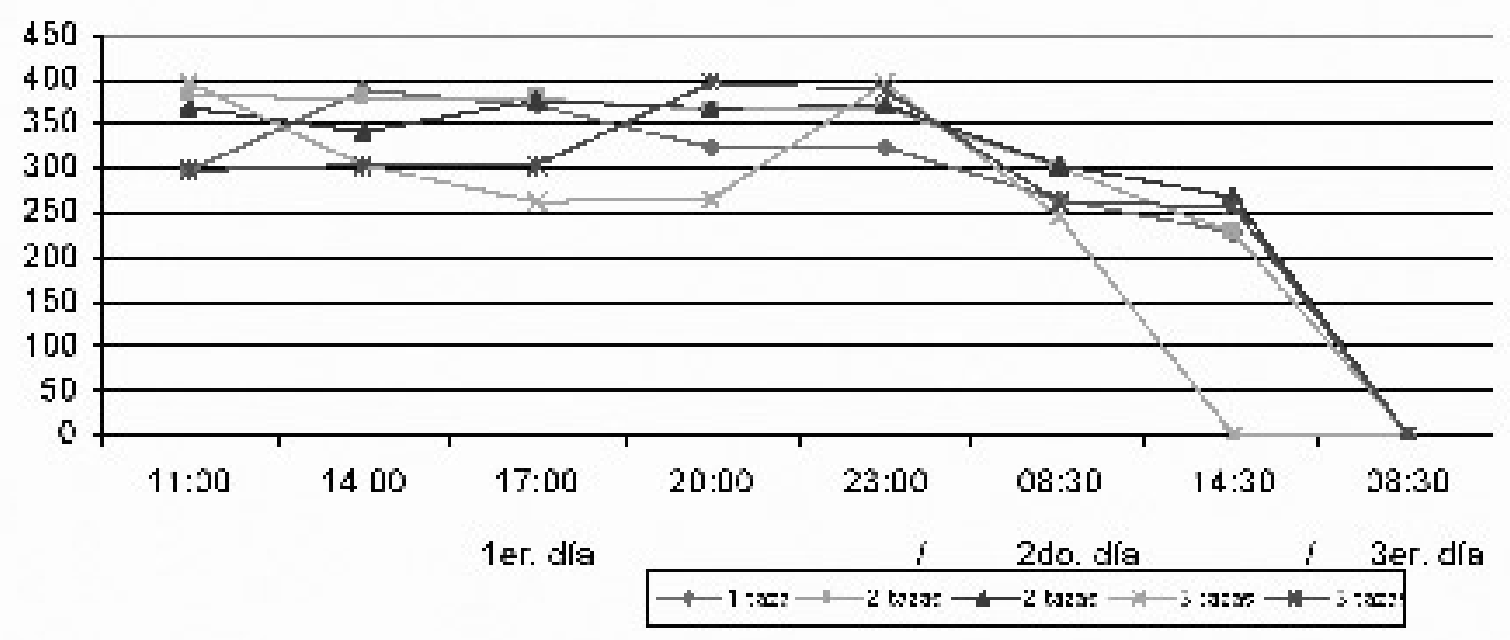

Figura 7. Concentración de benzoilecgonina en muestras de orina según horas de excreción (Personas que bebieron mate de coca en bolsitas)

horas para descender bruscamente después. En los otros dos casos (líneas de rombos y triángulos) durante 24 horas para después descender también bruscamente.

Finalmente, la figura número siete nos muestra fluctuaciones en la excreción de benzoilecgonina de varios voluntarios que bebieron mate de coca (una dos y tres tazas) que se hacen negativos a los tres días.

La duración de la positividad de benzoilecgonina en orina, proveniente de mate de coca, está en relación al metabolismo de la persona así como a su ingestión de líquidos, más que a la concentración o número de tazas consumidas (Ver línea de " $x^{\prime \prime}$ ).

\section{DISCUSIÓNY CONCLUSIONES}

En líneas generales, con los recursos y métodos actuales de investigación, solo si se toma la muestra de orina el mismo día en que se ingiere la coca o la cocaína, es posible distinguir al grupo consumidor de cocaína, en forma de clorhidrato y/o pasta base con el grupo de "acullicadores" y con aquel que consume hojas de coca en forma de mates. Podemos afirmar que las concentraciones bajas en orina (de 220 $\mathrm{ng} / \mathrm{ml}$ a $450 \mathrm{ng} / \mathrm{ml}$ ) corresponden a consumidores de cocaína, consumidores de mate y acullicadores. Cuando las concentraciones son más altas (de 451 $\mathrm{ng} / \mathrm{ml}$ a $560 \mathrm{ng} / \mathrm{ml}$ ) solo nos encontramos con acullicadores y gente que consumió cocaína. Cuando la concentración en orina está por encima de 561 ng/ $\mathrm{ml}$ el individuo consumió cocaína (en forma de pasta base, clorhidrato o pitillo).

A partir del segundo día de consumo de cualquiera de estas substancias, no es posible diferenciar uno del otro con claridad.

Es indiscutible el hecho de que tanto la hoja de coca seca, que se utiliza para fabricar mate en bolsitas, sola o acompañada de anís, manzanilla o cualquier otra substancia, produce los mismos metabolitos que la cocaína en forma de clorhidrato o pasta base. Es decir: Ecgonina, Metilecgonina, Benzoilecgonina y Norformilecgonina.

En el presente estudio, el grupo de los bebedores de mate en bolsitas tenían concentraciones superiores de benzoilecgonina en orina que el grupo de los bebedores de mate de hojas de coca, no obstante la evidente menor cantidad de hoja de coca en bolsitas (2 a 3 gr.) que la cantidad de hojas enteras remojadas (entre 6 a 15 enteras, es decir 5 a 10 gr.). El análisis posterior llevó a concluir que la hoja de coca triturada o molida, libera mayor cantidad de benzoilecgonina.

También existe diferencia fácilmente apreciable entre los tomadores de una taza de mate y aquellos que beben dos o más; pero no hay diferencia entre los que beben dos o tres tazas, pero nuevamente las diferencias pudieron ser establecidas de grupo a grupo, pero no de individuo a individuo.

La detección de benzoilecgonina en orina, sin importar si esta proviene del consumo de hoja de coca o de cocaína, se hace positiva entre los 30 a 45 minutos de la administrada (la hoja de coca, la cocaína y/o mate de coca) y permanece positiva por 72 horas. Si el consumidor de mate de coca bebe suficiente líquido, puede acelerar el tiempo de eliminación de 
benzoilecgonina. Por lo tanto, La duración de la positividad de benzoilecgonina en orina, está en relación al metabolismo de la persona así como a su ingestión de líquidos, más que a la concentración o cantidad de mate (o droga) consumida.

Por otra parte, si se conocen las consecuencias del consumo de cocaína en el organismo humano, el consumo de la hoja de coca en forma de mate y/o "acullico", a largo plazo, todavía no ha sido estudiado. La pregunta que surge es, si no es posible distinguir entre el consumidor de cocaína y el de hoja de coca como tal, ¿por qué los efectos orgánicos no son similares? Una hipótesis nos llevó a concluir que, en este tema, mucho tiene que ver la concentración de la cocaína ingerida como tal. Es decir: a mayor concentración de cocaína ingerida (pureza y cantidad) mayores y más rápidos efectos en el organismo humano y viceversa, aspecto que de todas maneras debe ser estudiado a profundidad. En cualquier caso, es bueno puntualizar que la cantidad de cocaína contenida en una, dos, diez o veinte hojas de coca, se miden en nanogramos, la ingerida en forma de pasta base o clorhidrato, en gramos. Finalmente, los efectos del "acullico" sobre el organismo humano a mediano y largo plazo, necesitan ser estudiados.

\section{REFERENCIAS}

Alcaraz F. (1989). Los Eslabones de la Droga. La Paz. Bolivia.

Instituto Boliviano de Biología de Altura (1997). Usos de la hoja de coca y salud pública. La Paz, Bolivia.

Zavaleta, A.; Castro de la Mata, R. (Sin fecha). Detección de metabolitos de cocaína en consumidores en mate de coca. Area de investigaciones CEDRO, Lima, Perú.

Universidad de Chile. Laboratorio de análisis antidoping de la " $U$ " impulsa incuestionable examen para detectar consumo de cocaína. Disponible en http://www.uchile. cl/undin2/actuales/noti2826.shtml (Consultado el 14/04/ 04). 
